Jeannie Braun, Simon Herm

\title{
Benutzerbefragung an der WLB Stuttgart im Dezember 2007
}

Im Rahmen der Projektphase (3. Dezember 2007 bis 15. Februar 2008) unseres integrierten Praxissemesters an der WLB führten wir als Projektarbeit vom 5. bis 18. Dezember 2007 eine Benutzerbefragung über die Lesesäle durch.

Ziel der Befragung war es, ein Stimmungsbild über den Haupt- und die Sonderlesesäle zu bekommen. Dabei sollten sowohl eine Momentaufnahme der Lesesaalbenutzer und deren Meinung über Zustand und Ausstattung des Lesesaals erstellt als auch Wünsche und Anforderungen der Benutzer an einen zukünftigen Lesesaal erfasst werden. Dies wurde in Hinblick auf einen möglichen Erweiterungsbau gewünscht. Darüber hinaus erhofften sich alle Beteiligten realistische Empfehlungen für kurz- und langfristige Verbesserungsmaßnahmen.

\section{Fragebogen}

Zusammen mit unserer Projektbetreuerin Frau Scholz einigten wir uns auf die für unser Vorhaben am besten geeignete Methode des fragebogengestützten Interviews. Dieses wurde auf rund 10-15 Minuten angesetzt. Die Befragung sollte sich an alle Benutzer richten, die innerhalb unseres zweiwöchigen Befragungszeitraums die Lesesäle nutzten. Laut Rechnung ergab sich bei 12 Öffnungstagen eine Stichprobengröße von 200 Personen, die wir letztlich mit 333 befragten Personen deutlich übertreffen konnten.

Der Fragebogen selbst setzte sich aus 19 Fragen in sechs Fragenkomplexen zusammen (Einordnung des Befragten, Nutzung, Literaturangebot, Technische Ausstattung, Arbeitsatmosphäre und Gesamtzufriedenheit). $\mathrm{Zu}$ jedem Fragenkomplex konnten Kommentare abgegeben werden.

\section{Durchführung}

Die Befragung selbst wurde an prominenter Stelle, durch ein Stehpult gekennzeichnet, im Eingangsbereich des Hauptlesesaals durchgeführt. Die prominente Stelle sorgte für ausreichende Aufmerksamkeit seitens der Benutzer. In einzelnen Fällen wurden Benutzer explizit angesprochen und gebeten, an der Befragung teilzunehmen. Die Befragung setzte sich aus einem angeleiteten Interview zusammen, welches anhand des Fragebogens mit den Benutzern durchgeführt wurde. Kommentare wurden in Stichworten codiert aufgenommen.

\section{Datenerfassung und -auswertung}

Die Erfassung der Daten aus den Fragebögen erfolgte mittels eines Barcodescanners und auf den Fragebogen zugeschnittenen Barcode-Sheets. Die Ant- 
worten wurden per Barcode eingescannt und direkt in eine hierfür erstellte PerlDatenbank übertragen. Aus dieser Datenbank erfolgte der Datenexport nach Microsoft Excel. Mit diesem Programm wurden die Antworten zusammengeführt, ausgewertet und visualisiert.

\section{Kurzüberblick Ergebnisse}

Als mit Abstand größte Gruppe unter den Benutzern stellten sich erwartungsgemäß die Studierenden $(53,3 \%)$ heraus, Platz zwei und drei belegten die Berufstätigen (15,9\%) und die wissenschaftlich Tätigen (13,8\%). 9,3\% sind Doktoranden oder Habilitanden. Die meistbenutzten Bestände sind Geschichte, Recht, Wirtschaftswissenschaften, Enzyklopädien, Sozialwissenschaften und Kunst. Fast jeder Zweite macht Gebrauch von den Zeitschriften und rund jeder Fünfte von den Tageszeitungen. Die Zeitungen als Mikrofilm oder gebunden werden von fast jedem Achten benutzt. Hauptnutzungszeiten sind nicht auf einen bestimmten Wochentag festzumachen. Ohnehin kommt jeder zweite Benutzer mehrmals die Woche. Vor- und Nachmittag sind so gut wie gleich stark benutzt (73,9\% bzw. 73,0\%). Rund jeder Zweite nutzt auch die Abendstunden im Lesesaal $(49,2 \%)$. Die Zufriedenheit mit den Öffnungszeiten spaltet die Nutzerschaft in zwei Lager: Rund der Hälfte reichen die derzeitigen Öffnungszeiten nicht aus; sie fordern vor allem längere Öffnungszeiten am Samstagnachmittag $(85,9 \%)$ und bis 22 Uhr an Werktagen (68,1\%). Die andere Hälfte ist mit den Öffnungszeiten durchweg zufrieden. Meistgenutzte Medien sind der Präsenzbestand mit $89,2 \%$ und der Magazinbestand mit 64,9\%. Auffallend hierbei ist vor allem auch der hohe Anteil an Nennungen von „eigene Materialien“ $(60,4 \%)$.

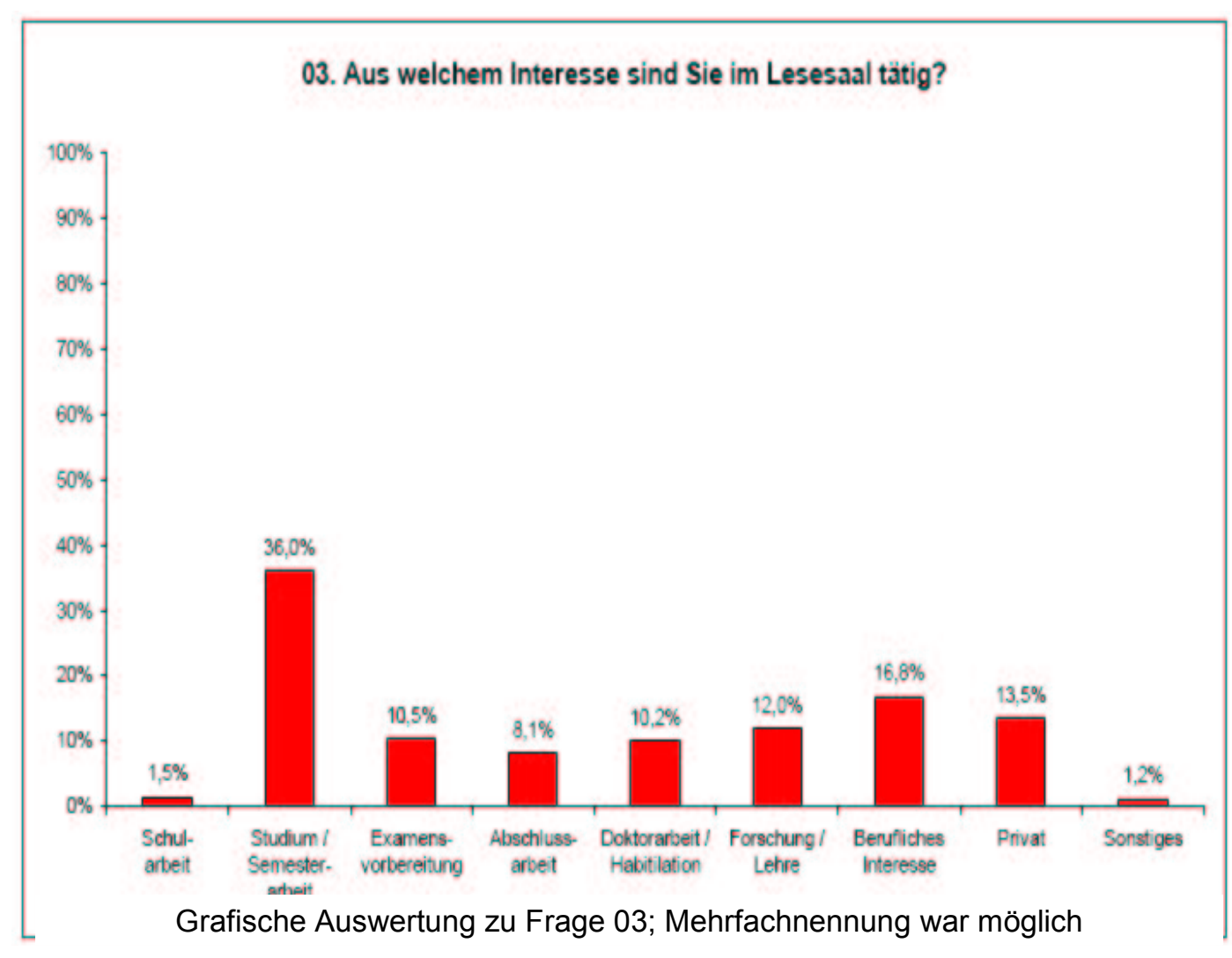




\section{Kurzüberblick Benotungen}

Im folgenden Abschnitt werfen wir einen kurzen Blick auf die von den Nutzern abgegebenen Benotungen.

Die Fragen zum Literaturangebot umfassten die Aktualität, Vollständigkeit, Verfügbarkeit und Orientierungshilfen in Fachbereich und Lesesaal. Aktualität $(2,19)$, Vollständigkeit $(2,45)$ und Verfügbarkeit $(2,53)$ wurden zwar zufriedenstellend bewertet, jedoch sind hier auf jeden Fall Verbesserungen möglich, z. B. durch die Bereitstellung von mehr Präsenzbestand im Lesesaal. Die Gesamtnote Literaturangebot beträgt 2,32.

Die technische Ausstattung wurde mit insgesamt 2,49 bewertet. Hier wurde nach der Zufriedenheit mit den Kopiergeräten, Scannern, Mikroformgeräten, dem WLAN sowie den Multimedia- und Katalog-PCs gefragt. Letztere fallen mit einem Durchschnitt von 1,91 positiv auf.

Bei der Arbeitsatmosphäre stechen die Aufstellung der Arbeitsplätze $(1,92)$ und die Größe der Arbeitstische $(2,07)$ hervor bei einem Durchschnittswert von 2,24 .

Abschließend wurden die Nutzer gebeten, ihrer Gesamtzufriedenheit mit den Lesesälen Ausdruck zu verleihen. Der Hauptlesesaal hat mit 2,06 abgeschnitten, der Zeitschriftenbereich mit 2,29, der Kunstlesesaal mit 1,86.

\section{Kommentare}

63\% der Befragten nutzten die Möglichkeit, ihre Bewertungen zusätzlich zu kommentieren. Die zehn häufigsten Nennungen waren:

„Zu kalt“, „Es zieht“, „Gespräche stören“, „Kopien zu teuer“, „Personal freundlich“, „Lesesaalschalter zu laut“, „Zu dunkel“, „Freies Internet im WLAN“, „Mehr Arbeitsplätze“ und „Arbeitsatmosphäre gut“.

\section{Schlussfolgerungen}

Die beiden vorherrschenden Kommentare „Zu kalt" und „Es zieht" waren vor allem durch die damaligen Baumaßnahmen im Haus und das Wetter bedingt. Darauf wurde bereits während der Befragungsphase durch entsprechende Temperaturregulierung kurzfristig reagiert.

Der häufig genannte Kommentar „Gespräche stören“ zeigt auf, dass sich viele Lesesaalbenutzer durch Gespräche gestört fühlen. Der Wunsch, in Gruppen mit Semester- und Präsenzbeständen arbeiten zu können, ist demnach bei vielen Nutzern vorhanden. Daher ist die Bereitstellung von Gruppenarbeitsplätzen dringend nötig und sinnvoll.

Weitere Geräuschquelle ist der Lesesaalschalter. Zukünftige bauliche Maßnahmen könnten hier Abhilfe abschaffen.

Im Fragenkomplex Nutzung wird der Wunsch nach verlängerten Öffnungszeiten 
am Samstag deutlich. Wünschenswert wäre, dies für den Erweiterungsbau zu berücksichtigen.

Die Tatsache, dass es in Stoßzeiten zu wahren Engpässen im Hauptlesesaal kommt, zwingt regelmäßig Benutzer dazu, auf die Sonderlesesäle auszuweichen, so dass sich viele Fachfremde in diesen wiederfinden (z. B. Juristen im Kunstlesesaal). Der Wunsch nach mehr Arbeitsplätzen kommt auch in den abgegebenen Kommentaren zum Ausdruck und sollte daher für den Erweiterungsbau berücksichtigt werden.

Angebote wie Scanner, WLAN und Multimedia-PC sind bei den Benutzern kaum bis gar nicht bekannt. Diese sollten besser kommuniziert werden, zum Beispiel durch neue Beschilderung oder Flyer.

Abgesehen von kleinen „Verbesserungsmöglichkeiten“ geben die Lesesäle der WLB aber auch genug Grund zur Zufriedenheit: Viele Benutzer beurteilen Arbeitsatmosphäre und Personal als gut beziehungsweise freundlich.

\section{Schlussbetrachtung}

Abschließend möchten wir unserer Projektbetreuerin Frau Scholz für die gute Zusammenarbeit danken sowie der EDV-Abteilung für die Ermöglichung der Datenerfassung per Barcodes. Dies hat die Datenerfassung wesentlich erleichtert. Ebenso danken wir allen Benutzern, die teilgenommen haben.

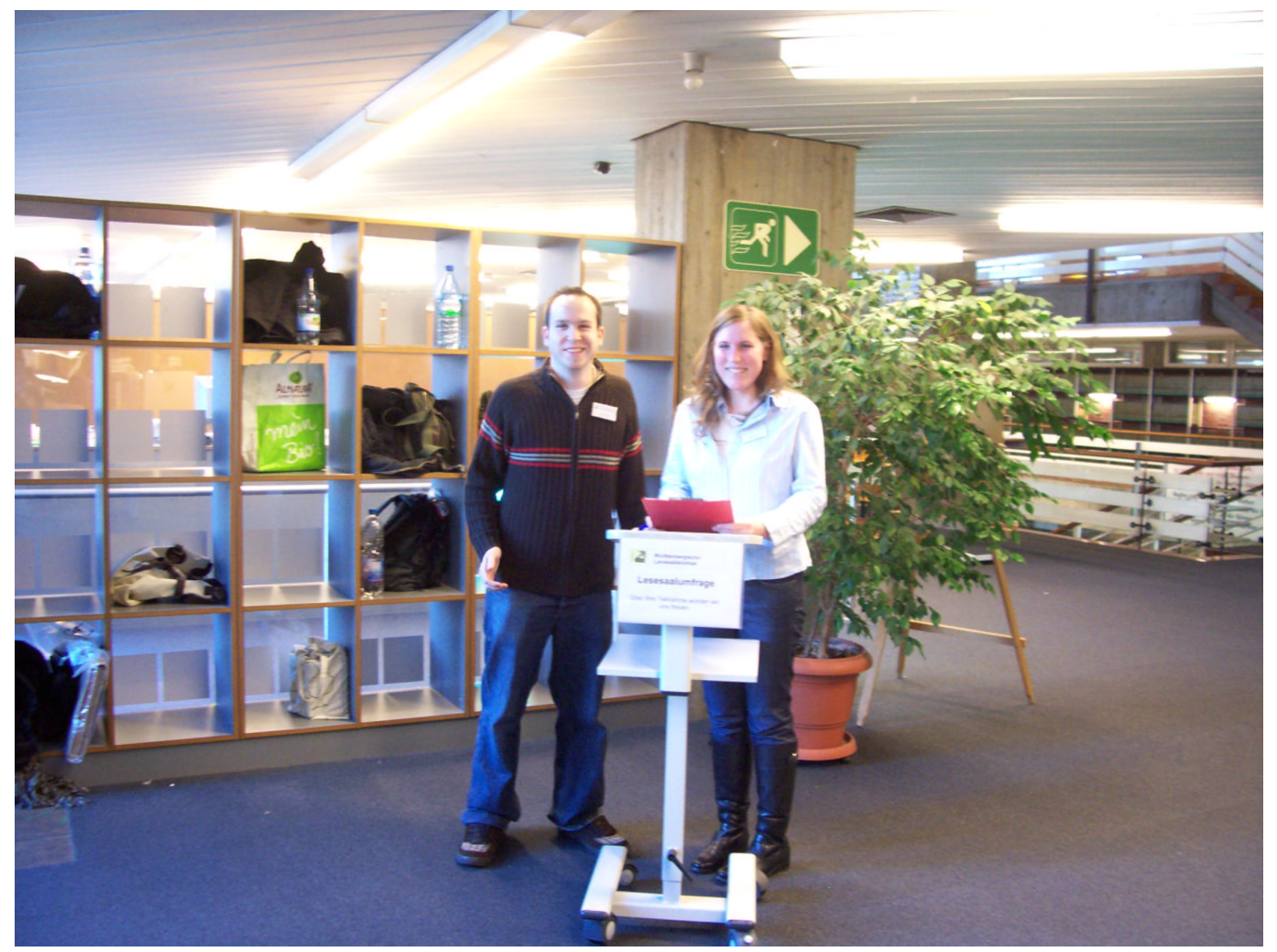

Projektteam Simon Herm und Jeannie Braun während des Befragungszeitraums 